Material and Methods We evaluated newborns who were born alive or dead with a birth weight of more than 500 grams and a gestational age over 22 weeks between January 2008 and December 2008 in our hospital.

Results In 2008, 3019 babies were born alive or dead with a birth weight of more than 500 grams and a gestational age over 22 weeks. Of these, 49 babies died in the perinatal period. Perinatal mortality rate was $36.7 \%$, stillbirth rate was $20.5 \%$, early neonatal mortality rate was $16.5 \%$. The causes of deaths according to a modified Wigglesworth classification were stillbirths, congenital malformations and prematurity and its complications, respectively.

Conclusion In our hospital, perinatal mortality rate has been declining in recent years. As a result, the some of neonatal deaths were due to complications of premature labor. Prevention of premature labor, sufficient antenatal maternal care and establishment of good delivery conditions to decrease neonatal infections and medical care after delivery could help to decrease neonatal mortality rates.

\section{NEONATAL MORBIDITY IN HYPERTENSIVE PREGNANCY}

doi:10.1136/archdischild-2012-302724.1369

DS Krstic. Neonatology, Health Centre Vranje, Vranje, Serbia

Research conducted at the Gynaecology-obstetrics and neonatology department of the Health Center Vranje in 2011./2012 included 80 mothers and 80 infants. The study included 40 mothers of patients with hypertensive syndrome in pregnancy and 40 mothers and infants in the control group.

Hypertension is registered in the $3-7 \%$ of pregnant women. The average age of pregnant women from the control group was 31 years and 26 years.

For mothers with hypertension, the labor was completed in 28 $(70 \%)$ by caesarean section and only in 12 (30\%) spontaneously, whereas in the control group leads spontaneously in 32 (80\%) mothers.

The average body weight of infants of mothers suffering from hypertensive syndrome was 2970 grams in the control group was 3235 grams.

Average Apgar score (cumulative score of cardiorespiratory function of newborns and nerve function) in the study group in the first minute was 6.55 , in the fifth 7.78, while in the control average Apgar score in the first minute is 7.67, and in the fifth minute 8.73.

Infants of mothers suffering from hypertensive syndrome in pregnancy have lower values of erythrocytes, $\mathrm{pH}, \mathrm{PO} 2$ and $\mathrm{PCO} 2$ greater value.

Duration of hospitalization, mothers suffering from hypertension of the newborn is longer.

The observed groups There were no maternal or fetal mortality.

\section{THE RELATIONSHIP BETWEEN PREGNANT MOTHER'S CONDITION AND NEONATAL MORTALITY RATE}

doi:10.1136/archdischild-2012-302724.1370

MS Kosim, NA Nenden, B Saad, AR Farid. Department of Pediatrics, Faculty of Medicine Diponegoro University - Dr. Kariadi Hospital, Semarang, Indonesia

Background Neonatal mortality is still a problem around the world. The rate is varies from country to country. Mother's condition was associated with the outcome of conception and mortality rate.

Objective To determine the relationship between mother's condition and neonatal mortality rate.

Methods/design Case control study. Case group was 40 mothers of neonates who died in high risk neonatal ward Dr. Kariadi Hospital Semarang Indonesia within period of January to December 2011 who fulfilled inclusion criteria. Control group was 40 mothers of neonates who survived. Mother's condition that was studied included mother's age, parity, gestational age, antenatal care, mother's infection, diabetic mother, severe anemia, preeclampsia, eclampsia, hypertension, heart disease, and antenatal bleeding. Data was taken from medical records. Statistical analyses used $\mathrm{X}^{2}$ and logistic regression.

Result Between groups respectively: age $>30$-year old, has OR 0.70; 95\% CI $0.27-1.82$ ). multiparity (OR $1.22 ; 95 \%$ CI $0.51-2.96$ ). preterm, (OR 2.78; 95\% CI 1.12-6.89). ANC, (OR 0.87; 95\% CI $0.31-$ 2.44). Mother's infection, (OR 0.29; 95\% CI 0.05-1.58). diabetic mother, (OR 4.33; 95\% CI 0.46-40.61). severe anemic, d (OR 2.29; 95\% CI 1.76-2.98). preeclampsia mothers, (OR 0.23; 95\% CI 0.02 2.16). eclampsia mothers,(OR 2.71; 95\% CI 0.49-14.90). hypertension mothers, (OR 4.33; 95\% CI 0.46-40.60). heart disease (OR 2.02; $95 \%$ CI 1.62-2.53). antenatal bleeding (OR 2.33; 95\% CI 1.78-3.05).

Conclussion Gestational age (preterm), severe anemia, and antenatal bleeding were associated with neonatal mortality, where gestational age as the major risk factor.

\section{NEONATAL MORTALITY REVIEWS IN A TERTIARY NEONATAL UNIT IN UK: AN USEFUL LEARNING EXPERIENCE}

doi:10.1136/archdischild-2012-302724.1371

S Mitra, K Beardsall, H O'Reilly. Neonatal Unit, The Rosie Hospital, Cambridge University Hospitals NHS Foundation Trust, Cambridge, UK

Background and Aims Neonatal care has developed dramatically over the last few decades. In spite of this, the neonatal mortality remains high. As clinicians we strive to deliver high quality care and by reviewing the care in all neonatal deaths we hope to identify the avoidable risk factors, areas of good practice and areas for improving care and optimising future service delivery.

Methods Patient notes of all neonates died over one year ( $1^{\text {st }}$ Jan 2010-31 $1^{\text {st }}$ Dec 2010) period in a tertiary neonatal unit in UK were reviewed by members of a multidisciplinary team. Reviews were performed using a structured format assessing all areas of care including resuscitation, clinical management, transfer, communication and documentation.

Results 1018 infants were admitted to the neonatal unit, 878 infants were in-born and 140 infants were ex-utero transfers. Most deaths (14 infants $(1.3 \%)$ ) occurred within first seven days of life, 8 infants $(0.7 \%)$ died in the late neonatal period and 5 infants $(0.5 \%)$ died after 28 completed days.

Several areas of good practices were identified, including evidence of good multi-disciplinary team working. Key themes were identified as areas for improvement including documentation and continuity of care at consultant level for infants with complex needs and longer stay. An annual report summarising all cases and recommendations was produced.

Conclusion Mortality case reviews are an important source of learning. In order to successfully influence the service development these reviews must be structured, include input from a multi-disciplinary team and result in specific and achievable recommendations.

\section{2 \\ RISK FACTORS OF PERINATAL MORTALITY: MOROCCAN DATA}

doi:10.1136/archdischild-2012-302724.1372

'A Barkat, 'N Sabir, ${ }^{2} \mathrm{M}$ Kabiri. 'Faculté de Médecine et de Pharmacie de Rabat; Université Souissi; ${ }^{2}$ CRECET; Faculté de Médecine et de Pharmacie de Rabat, Rabat, Morocco

Objective The objective of this study was to analyze the main factors of perinatal mortality. 
Study Design This prospective, descriptive and analytical study was conducted in the maternity unit Souissi - Rabat over a one year period from January 2010 to December 2010. Obstetric and perinatal records are used in this study. A sheet filled out for each newborn comprising the socio-demographic, obstetric, nursery data, monitoring and surveillance of pregnancy, delivery as well as neonatal data, the group of dead and alive patients were compared and analyzed.

Results There were a collection of 1000 births, 971 were alive and 29 dead on which 9 were stillborn and 20 died during the first 24 hours of life. There was a singleton pregnancy in 988 cases $(98.8 \%)$, twins in 11 cases $(1.1 \%)$ and triple in 1 case $(0,1 \%)$. The statistical analysis was used to determine the prognostic factors significantly associated with the occurrence of perinatal mortality namely the Apgar $<7$ in $1 \mathrm{~min}$ and in 5 minutes $(p<0.001)$, the low birthweight $(p<0.001)$, the low gestational age $(p<0.001)$, dosage of the medication or plants during pregnancy $(p=0.01)$, the presence of congenital abnormalities $(p<0.001)$ and the multiplicity $(p=0.002)$.

\section{THE RELATIONSHIP BETWEEN BIRTHWEIGHT AND NEONATAL MORTALITY}

doi:10.1136/archdischild-2012-302724.1373

S Gatot-Irawan, A Nur Radityo, S Ariawan, MS Kosim, ER Arsita. Department of Pediatrics, Faculty of Medicine Diponegoro University - Dr. Kariadi Hospital, Semarang, Indonesia

Background Neonatal mortality rate plays a role for almost 40 per cent of under-five child mortality, around the world. An understanding about the factors related to neonatal mortality is important to prevent neonatal deaths. Birth weight was known as one of a risk factors and many studies has been conducted.

Objective To determine the relationship between birthweight and neonatal mortality.

Methods We conducted a hospital-based case control in Dr. Kariadi Hospital Semarang, Indonesia. Data were taken from medical records of babies who fulfilled inclusion criteria and admitted from January 2010 until December 2011. Neonatal mortality was defined as a death of neonates (until 28 days). Birth weight was determined at birth with same scale and categorized in to some caregories. Statistical analyses used: $\mathrm{X}^{2}$ and logistic regression.

Results We obtained 278 babies (18\% from totally babies) as a neonatal death as a Case Group and 280 babies as Control group from level 2 ward Kariadi Hospital. Between two groups respectively: Very low birth weight babies has (OR 0.5; 95\% CI; 0.2-0.8). Low birth weight babies has (OR 1.5; 95\% CI; 1.1-2.2), and appropriate birth weight has (OR 1.1; 95\% CI; 0.8-2.5).

Conclusion Low birth weight babies was a risk factor for neonatal mortality.

\section{RISK FACTORS FOR NEONATAL MORTALITY RATE}

doi:10.1136/archdischild-2012-302724.1374

MS Kosim, A Nur Radityo, T Taufan-Prasetya, S Gatot-Irawan. Department of Pediatrics, Faculty of Medicine Diponegoro University - Dr. Kariadi Hospital Semarang Indonesia, Semarang, Indonesia

Background Neonatal mortality rate plays a role for almost 40 per cent of under-five child mortality, globally. An understanding about the factors related to neonatal mortality is important to guide the development of focused and evidence-based health interventions to prevent neonatal deaths.

Objective To determine risk factor for neonatal mortality.

Methods We conducted a hospital-based case-control study in Kariadi Hospital Semarang, Indonesia. Data was taken from medical records of babies who fulfilled inclusion criteria and admitted from January 2010 until December 2011. Neonatal mortality was defined as a death of neonates (until 28 days). Risk factors were studied included: birth weight, gestation period, sepsis, type of delivery, length of stay (LOS) Statistical analyses used: $\mathrm{X}^{2}$ and logistic regression.

Results We obtained 278 (18\% from totally babies who admitted) neonatal deaths as a Case Group and 280 babies as Control group from level 2 ward Kariadi Hospital. We found between two groups prematurity has $(\mathrm{OR}=1.6$; $95 \% \mathrm{CI}: 1.1-2.6)$, low birth weight in prematurity $(\mathrm{OR}=2.0 ; 95 \% \mathrm{CI}: 1.2-3.5)$ and sepsis in prematurity $(\mathrm{OR}=2.2$; 95\% CI:1.1-4.2) while other risk factors (birth weight, type of delivery, LOS) have lower points.

Conclusion Sepsis in prematurity was a risk factor for neonatal mortality.

\section{EARLY DIAGNOSTICS OF NECROTIZING ENTEROCILITIS IN NEWBORN INFANTS UNDERWENT PERINATAL ASPHYXIA}

doi:10.1136/archdischild-2012-302724.1375

A Haciyeva. Scientific Research Institute of Pediatrics named after of K.J. Farajova, Baku, Azerbaijan

Background and Aim Defensins are antimicrobial peptides against gram positive and gram negative bacteria, fungus and viruses. The goal of the study is to reveal diagnostic fecal markers of necrotizing enterocolitis (NEC) among the various gestational age children who underwent perinatal asphyxia.

Methods The 95 newborn children have been included to study. Main group consisted of 960-4210 g birth weighing 69 infants with structural pathologies of central nervous system. Main group were classified in two subgroups: 40 preterm newborns (gestational age 30-37 weeks) with structural pathologies of central nervous system and 29 term babies with $38-40$ weeks of gestational age. 26 children (14 premature and 12 in term infants) were included in control group. Stool for analyses was taken in 3-rd, 7-th, 15-th and 30-th days of life. The concentration of human $\beta$-defensin-2 (HBD-2) was determined by immune-enzyme analysis (ELISA Kit Immune diagnostic, Bensheim, Germany).

Results In premature babies NEC development associated with the 246.2- $257.5 \mathrm{ng} / \mathrm{g}$ on 3-rd day; 173.7-206.9 ng/g on 7-th day; 161.5$188.9 \mathrm{ng} / \mathrm{g}$ on 15-th day; $155.2-167.4 \mathrm{ng} / \mathrm{g}$ on 30-th day of HBD-2 concentration. In term babies NEC developed in $246.2-268.5 \mathrm{ng} / \mathrm{g}$ on 3-rd day; 166.9-255ng/g on 7-th day, 161.5-226 ng/g on 15-th day; $155.2-208 \mathrm{ng} / \mathrm{g}$ on 30-th day of HBD-2 concentration.

Conclusion Thus, definition of fecal HBD-2 in dynamics at newborn children underwent to perinatal asphyxia allows to diagnose NEC at the initial stage of development which gives the base to begin in time treatment.

\section{PROBIOTICS FOR PREVENTION OF NECROTIZING ENTEROCOLITIS IN PRETERM VLBW NEONATES- SYSTEMATIC REVIEW OF RANDOMISED CONTROLLED TRIALS. (UPDATE 2011)}

doi:10.1136/archdischild-2012-302724.1376

1,2G Deshpande, ${ }^{3,4} \mathrm{~S}$ Rao, ${ }^{4,5}$ S Patole, ${ }^{6} \mathrm{M}$ Bulsara. ${ }^{1}$ Neonatology, Nepean Hospital Sydney; ${ }^{2}$ Nepean Clinical School, University of Sydney, Kingswood; ${ }^{3}$ Neonatology, Princess Margaret Hospital for Children, Perth, NSW; ${ }^{4}$ Centre for Neonatal Research and Education, School of Womens and Infants Health, University of Western Australia, Perth, WA; ${ }^{5}$ Neonatology, King Edward Memorial Hospital, Perth, NSW; ${ }^{6}$ University of Notre Dam, Perth, WA, Australia

Background Systematic reviews indicate significantly lower risk of NEC and mortality, and shorter time to full feeds after probiotic supplementation in preterm (Gestation $<33$ weeks) VLBW neonates. ${ }^{1-3}$ Few more RCTs have been published since then. 